\title{
Clinical and Imaging Analysis of a Cerebellar Watershed Infarction
}

\author{
Mei Zheng, Aping Sun, Qingli Sun, Hui Zhang, Dongsheng Fan* \\ Department of Neurology, Peking University Third Hospital, Beijing, China \\ Email: ${ }^{*}$ dsfan@sina.com
}

Received 1 February 2015; accepted 10 March 2015; published 11 March 2015

Copyright (C) 2015 by authors and Scientific Research Publishing Inc.

This work is licensed under the Creative Commons Attribution International License (CC BY). http://creativecommons.org/licenses/by/4.0/

(c) (i) Open Access

\section{Abstract}

Objective: To investigate the characteristics of vascular lesions in patients with a cerebellar watershed infarction. Methods: Clinical data from 178 cases of cerebellar infarction were collected with magnetic resonance imaging (MRI) scan results, including diffusion weighted imaging (DWI), a magnetic resonance angiography (MRA), and computed tomography angiography (CTA). The cases were divided into cerebellar watershed and non-watershed infarctions based on lesion location, which was revealed by DWI. Forty-two cases met the criteria for a cerebellar watershed infarction. Based on the MRA/CTA results, the vertebrobasilar artery stenoses were divided into four categories (i.e., intracranial, extracranial, combined, and no detectable stenosis) to compare the vascular lesion characteristics from patients with a cerebellar watershed infarction and patients with a non-watershed infarction. Results: Patients with cerebellar watershed infarcts presented mild symptoms at onset and had a favorable prognosis. However, $90.5 \%$ of these patients had a vascular stenosis, which was higher than for patients with a cerebellar non-watershed infarction (74.3\%). The four types of vascular lesions, intracranial, extracranial, combined, and no detectable stenosis, were $14.3 \%, 52.4 \%, 23.8 \%$, and $9.5 \%$ in patients with a cerebellar watershed infarction and $33.8 \%, 16.2 \%, 24.3 \%$, and $25.7 \%$ in patients with a non-watershed cerebellar infarction, respectively. Conclusion: Although patients with cerebellar watershed infarcts often had comparatively benign clinical manifestations and prognoses, such patients also had a high stenosis prevalence in major blood vessels, especially the extracranial segment in the vertebral artery, which required early intervention and treatment.

\section{Keywords}

Watershed Infarction, Cerebellum, Magnetic Resonance Imaging, Magnetic Resonance Angiography, Computed Tomography Angiography

\footnotetext{
${ }^{*}$ Corresponding author.
} 


\section{Introduction}

A cerebellar watershed infarction is a rare type of brain infarction. In 1993, Amarenco et al. [1] reported 47 patients with very small (border zone) cerebellar infarcts and revealed that large or pial artery disease rather than systemic hypotension was the main vascular lesion. However, the small cerebellar infarcts were recommended a new classification system based on topography in recent study [2]. A limited number of studies on cerebellar watershed infarction have been published, and the pathogenesis, clinical manifestations, location of vascular lesions, and prognosis for this disease are still unclear. In our study, we collected 42 cases with a cerebellar watershed infarction and analyzed the clinical and imaging features to understand the corresponding vascular pathogenesis that was helpful to the therapy and secondary prevention of this rare disease.

\section{Subjects and Methods}

\subsection{Research Subjects}

We collected 178 new cases with a cerebellar infarction that were admitted to the Department of Neurology at the Peking University Third Hospital from January 2008 to June 2011; the patients met the diagnostic criteria listed in the China Guideline for diagnosis and treatment of acute ischemic stroke (2010) [3]. The inclusion criteria required the following: 1) infarction involved with the cerebellum, which was confirmed as an acute cerebellar infarction through DWI; 2) magnetic resonance imaging (MRI), diffusion weighted imaging (DWI), and magnetic resonance angiography/computed tomography angiography (MRA/CTA) examinations (including head and neck blood vessels) completed within 1 week of onset; 3) blood examinations, including routine blood test, blood coagulation, fibrinogen, homocysteine, blood glucose, blood lipids, electrolytes, liver and kidney function, as well as an electrocardiogram and echocardiogram, completed within 1 week of onset. The exclusion criteria were as follows: a) patients with a cardiogenic cerebral embolism, including atrial fibrillation, myocardial infarction within 3 weeks, dilated cardiomyopathy, rheumatic heart disease, and a ventricular aneurysm; and b) patients currently receiving thrombolytic or anticoagulant therapy. Our institutional review board approved this study, but patient informed consent was not required because this study was retrospective. All the patients received the necessary treatment including thrombolysis, anti-platelet, or anticoagulin according to individual pathogenesis.

\subsection{Baseline Data Collection}

We recorded the patients' age, gender, stroke risk factors (hypertension, hyperlipidemia, diabetes, hyperhomocysteinemia, and smoking), the time interval from onset to hospital admission, blood pressure upon hospital admission, and NIHSS scores upon hospital admission and 1 week after admission.

\subsection{Imaging Examination}

MRI, DWI, and head and neck MRA/CTA examinations were completed within 1 week of onset. Patients were divided into watershed and non-watershed infarction cases based on the cerebellar infarction location. Cerebellar watershed infarctions included infarctions in the following watershed zones: between the superior cerebellar and posterior inferior cerebellar arteries; between the superior cerebellar and anterior inferior cerebellar arteries; and between the anterior inferior cerebellar and posterior inferior cerebellar arteries. Cerebellar non-watershed infarctions included infarctions in the superior cerebellar, anterior inferior cerebellar, and posterior inferior cerebellar arteries. The patients' head and neck blood vessels were examined using MRA or CTA. The results were interpreted by two clinicians without the patients' clinical data, and patients who had $>50 \%$ of blood vessels with stenosis were counted. The stenoses in the vertebrobasilar arterial system were divided into four types based on the stenosis location: intracranial stenosis, extracranial stenosis, combined stenosis, and no detectable vascular stenosis.

\subsection{Statistical Analysis}

The SPSS 16.0 software package was used for statistical analysis. The quantitative data were expressed as the mean $\pm \mathrm{s}$; the groups were compared using a t-test with two independent samples; the categorical data were compared using a $\chi^{2}$ test for two groups; and $\mathrm{P}<0.05$ indicated a statistically significant difference. 


\section{Results}

\subsection{Patients' Baseline Characteristics}

We included 178 cases with a cerebellar infarction, and 42 cases (24\%) had cerebellar watershed infarctions. No significant differences in age, sex, or risk factors of stroke were found between the two groups of patients. However, the time interval from onset to hospital admission was significantly longer for the watershed infarction group compared with the non-watershed infarction group $(\mathrm{P}<0.01)$. For blood pressure at hospital admission, no significant difference was observed in the baseline diastolic blood pressure between the watershed infarction and non-watershed infarction groups, but the baseline systolic blood pressure was much lower in the watershed infarction group compared with the non-watershed infarction group $(\mathrm{P}<0.01)$. When admitted to the hospital, the NIHSS score for the watershed infarction group was significantly lower compared with the nonwatershed infarction group ( $\mathrm{P}<0.01$ ) (Table 1). After one week, the NIHSS score for the watershed infarction group decreased from $2.33 \pm 1.69$ to $1.64 \pm 1.19(\mathrm{t}=2.166, \mathrm{P}=0.033)$, and the non-watershed infarction group's score increased from $4.21 \pm 2.39$ to $4.88 \pm 4.44(\mathrm{t}=1.593, \mathrm{P}=0.112)$, which indicates that a cerebellar watershed infarction yields a good short-term prognosis.

\subsection{Cerebellar Infarction Locations}

Among the 42 patients with a cerebellar watershed infarction, 32 patients $(76.2 \%)$ had a cerebellar watershed infarction in the watershed zone between the superior cerebellar and posterior inferior cerebellar arteries (Figure 1); 8 cases (19.0\%) had a cerebellar watershed infarction in the watershed zone between the anterior inferior cerebellar and posterior inferior cerebellar arteries (Figure 2); and 2 cases (4.8\%) had a cerebellar watershed infarction in the watershed zone between the superior cerebellar and anterior inferior cerebellar arteries (Figure 3).

Among the 136 patients with cerebellar non-watershed infarctions, 67 cases (49.3\%) had a superior cerebellar artery infarction; 22 cases (16.2\%) had an anterior inferior cerebellar artery infarction; and 47 cases (34.6\%) had a posterior inferior cerebellar artery infarction.

Table 1. Patients' baseline characteristics.

\begin{tabular}{|c|c|c|c|c|}
\hline Item & $\begin{array}{l}\text { Watershed infarction } \\
\qquad(\mathrm{n}=42)\end{array}$ & $\begin{array}{l}\text { Non-watershed infarction } \\
\qquad(\mathrm{n}=136)\end{array}$ & t value $/ \chi^{2}$ value & $\mathrm{P}$ value \\
\hline Age (year, $\bar{x} \pm \mathrm{s}$ ) & $68.2 \pm 9.8$ & $67.6 \pm 9.2$ & 0.702 & 0.338 \\
\hline Gender (male/female) & $96 / 40$ & $31 / 11$ & 0.163 & 0.687 \\
\hline \multicolumn{5}{|l|}{ Stroke risk factors (n, \%) } \\
\hline Hypertension & $16(38.1)$ & $63(46.3)$ & 0.880 & 0.348 \\
\hline Hyperlipidemia & $12(28.6)$ & $40(29.4)$ & 0.011 & 0.917 \\
\hline Diabetes & $8(19.0)$ & $37(27.2)$ & 1.131 & 0.288 \\
\hline Hyperhomocysteinemia & $12(28.6)$ & 35 (25.7) & 0.133 & 0.716 \\
\hline Smoking & $10(23.8)$ & $46(33.8)$ & 1.492 & 0.222 \\
\hline $\begin{array}{l}\text { Time interval from onset to hospital } \\
\text { admission (h, } \bar{x} \pm \mathrm{s})\end{array}$ & $36.69 \pm 20.81$ & $16.69 \pm 8.23$ & 9.164 & 0.000 \\
\hline $\begin{array}{l}\text { Baseline systolic blood pressure } \\
\text { (mmHg, } \bar{x} \pm \mathrm{s})\end{array}$ & $141.81 \pm 25.84$ & $161.06 \pm 13.45$ & 4.538 & 0.000 \\
\hline $\begin{array}{l}\text { Baseline diastolic blood pressure } \\
\qquad(\mathrm{mmHg}, \bar{x} \pm \mathrm{s})\end{array}$ & $83.36 \pm 15.20$ & $86.31 \pm 9.92$ & 1.470 & 0.143 \\
\hline NIHSS score (score, $\bar{x} \pm \mathrm{s}$ ) & & & & \\
\hline On hospital admission & $2.33 \pm 1.69$ & $4.21 \pm 2.39$ & 4.729 & 0.000 \\
\hline 1 week later & $1.64 \pm 1.19$ & $4.88 \pm 4.44$ & 4.660 & 0.000 \\
\hline
\end{tabular}



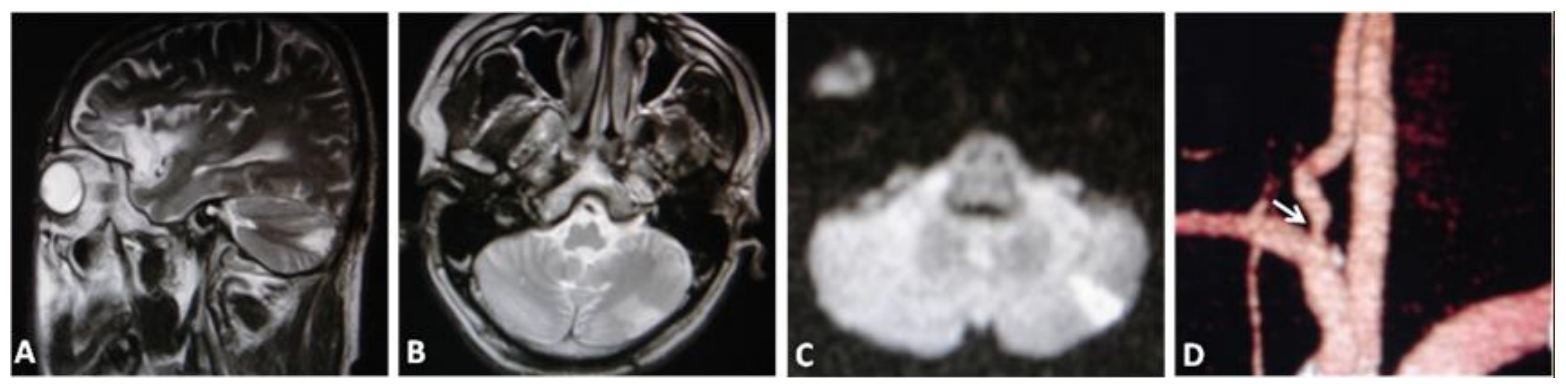

Figure 1. An infarction in the watershed zone between the superior cerebellar and posterior inferior cerebellar arteries. (A) Case 1, a 70-year-old male with sudden dizziness, unsteady gait, and failure to walk on toes or heels during a physical examination. The sagittal T2-weighted image showed that the lesion was located at the border between the superior cerebellar and posterior inferior cerebellar arteries; the lesion was wedge-shaped with the base proximal to the cerebellar surface, and the tip pointed to the deep cerebellum; (B) The axial T2-weighted image revealed the wedge-shaped lesion was located in the plane with the lower end of the pons; (C) DWI indicated that the lesion was an acute brain infarction; (D) A CTA of the neck vessels showed moderate stenosis in the initial segment of the left vertebral artery (white arrow).
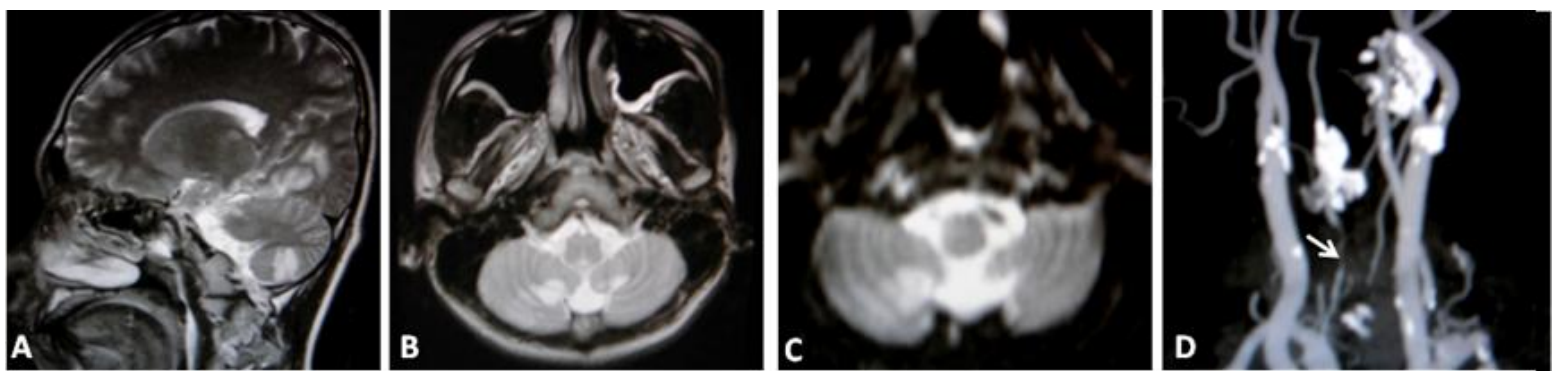

Figure 2. An infarction in the watershed zone between the anterior inferior cerebellar and posterior inferior cerebellar arteries. (A) Case 2, a 54-year-old male with sudden dizziness, unsteady gait, dysarthria during a physical examination, and right limb ataxia. The sagittal T2-weighted image showed that the lesion was located at the border between the anterior inferior cerebellar and the posterior inferior cerebellar arteries, and the lesion was wedge-shaped; (B) The axial T2-weighted image revealed the wedge-shaped cerebellar lesion was in the plane with the medulla oblongata; (C) DWI showed that the lesion was an acute brain infarction; (D) An enhanced MRA of the neck vessels revealed a severe stenosis in the right vertebral artery at the C5-C6 level (cervical 5 and 6) (white arrows).
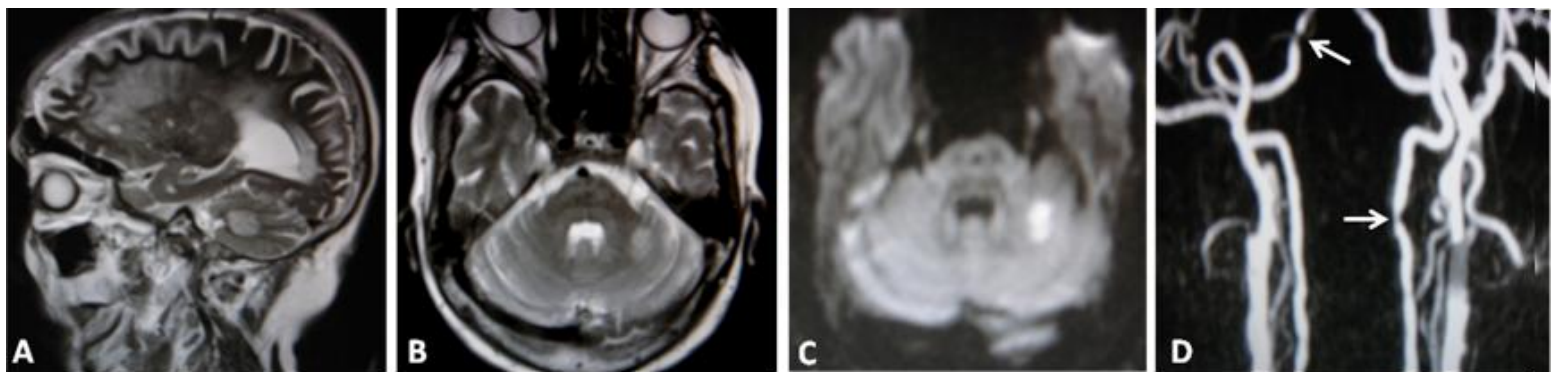

Figure 3. An infarction in the watershed zone between the superior cerebellar and anterior inferior cerebellar arteries. (A) Case 3, a 72-year-old female with sudden dizziness, slurred speech, ataxic dysarthria with a prosodic deficit during a physical examination, and left limb ataxia. The sagittal T2-weighted image showed that the lesion was located at the border between the superior cerebellar and anterior inferior cerebellar arteries; (B) The axial T2-weighted image showed that the lesion was located deep in the cerebellum in the plane with the pons; (C) DWI showed that the lesion was an acute brain infarction; (D) An enhanced MRA of the neck vessels revealed a moderate stenosis in the left vertebral artery at the C3 level and severe stenosis in the intracranial segment of the right vertebral artery (white arrows).

\subsection{The Relationship between the Cerebellar Infarction Location and Blood Vessels}

The prevalence of vascular stenosis in cerebellar watershed infarction patients was $90.5 \%$ (38/42), which was significantly higher than the $74.3 \%(101 / 136)$ rate of vascular stenosis in cerebellar non-watershed infarction patients $\left.\chi^{2}=4.929, \mathrm{P}<0.05\right)$. In addition, the prevalence of stenosis in the extracranial segment of the verte- 
brobasilar system was significantly higher in patients with a cerebellar watershed infarction than for patients with a cerebellar non-watershed infarction $(\mathrm{P}<0.01)$. The prevalence of intracranial stenosis and no detectable vascular stenosis were significantly lower in patients with a cerebellar watershed infarction than in the cerebellar non-watershed infarction group $(\mathrm{P}<0.05)$, and the combined stenosis prevalence was not significantly different between the two groups $(\mathrm{P}>0.05)$ (Table 2).

\section{Discussion}

We performed imaging for the 178 admitted cases with a cerebral infarction. For certain patients with a smaller infarct size, the infarct area location was not within an artery that supplied blood to the cerebellum, but it was localized at the border between two arteries, which is indicative of a cerebellar watershed infarction. Cerebellar watershed infarctions can be divided into two types; one has wedge-shaped lesions that affect the cortex, and the other is located deep in the cerebellum. A cerebellar watershed infarction is rarely observed in clinical practice, and its incidence has not been reported. In our study, $24 \%$ of patients with a cerebellar infarction had watershed infarctions. However, because patients with a cerebellar watershed infarction have significantly less severe symptoms compared with other cerebellar infarction patients, certain cerebellar infarction patients might have died before a vascular examination. Therefore, the cerebellar watershed infarction incidence may be lower than this value.

Current studies on watershed infarction pathogenesis are based on a watershed infarction of the carotid system [4]-[6]. Because a watershed infarction has been observed in patients with acute hypotension from abnormal cardiac and pulmonary function [7] [8], early studies suggested that pathogenesis was related to low perfusion conditions. Ultrasonographic and autopsy studies [9] [10] revealed that watershed infarct areas comprise many microemboli, and most of the microemboli are derived from atherosclerotic plaques in the internal carotid and middle cerebral arteries, which suggests a novel microembolism mechanism. It has also been suggested [11] that hemodynamic abnormalities induced by aortic stenosis could cause decreased local cerebral perfusion pressure, and microemboli accumulate in the vascular periphery, which cannot easily be washed away and generate an infarction in the watershed area. Further studies have classified watershed infarctions in the internal carotid artery system as an internal watershed infarction (or subcortical watershed infarction) and a cortical watershed infarction. Modern imaging studies suggest that an internal watershed infarction is primarily caused by hypoperfusion [12], while microembolism is the primary cause of a cortical watershed infarction [13]. For the relationship between watershed infarction and arteriosclerosis, studies outside China have suggested that an internal watershed infarction is symptomatic of an internal carotid artery stenosis or occlusion, whereas domestic research indicates that the most common cause of a cerebral watershed infarction is a cerebral artery stenosis or occlusion followed by an internal carotid artery stenosis or occlusion. Yong et al. [14] found that $76.3 \%$ of internal watershed infarction patients had a severe (stenosis $>75 \%$ ) internal carotid artery or middle cerebral artery stenosis, while approximately $52.3 \%$ of cortical watershed infarction patients had a severe carotid artery or middle cerebral artery stenosis. Given the low incidence of cerebellar watershed infarction, no in-depth studies on its pathogenesis have been performed. Our study focused on vascular anomalies in patients with a cerebellar watershed infarction and explored its pathogenesis.

In our study, the prevalence of a vascular stenosis in cerebellar watershed infarction patients was $90.5 \%$, which is significantly higher than for non-watershed cerebellar infarction patients. Although they focused on different arterial systems, our results revealed a significantly higher rate of vascular stenosis compared with Yong et al. [14] because we set the criteria for vascular stenosis at $>50 \%$. For cerebellar watershed infarction cases, the proportion with a stenosis in the vertebrobasilar extracranial segment was the highest (52.4\%),

Table 2. Comparison of the vertebrobasilar lesion locations between cerebellar watershed and non-watershed infarctions.

\begin{tabular}{ccccc}
\hline & Watershed infarction $(\mathrm{n}=42)$ & Non-watershed infarction $(\mathrm{n}=136)$ & $\chi^{2}$ value & P value \\
\hline Intracranial stenosis (n, \%) & $6(14.3)$ & $46(33.8)$ & 5.924 & 0.015 \\
Extratracranial stenosis (n, \%) & $22(52.4)$ & $22(16.2)$ & 22.603 & 0.000 \\
Combined stenosis (n, \%) & $10(23.8)$ & $33(24.3)$ & 0.004 & 0.952 \\
No detectable stenosis (n, \%) & $4(9.5)$ & $35(25.7)$ & 4.929 & 0.026 \\
\hline
\end{tabular}


which was higher than for extracranial vascular stenosis in non-watershed infarction cases. For non-watershed infarction cases, an intracranial vascular stenosis was more common (33.8\% of non-watershed infarction cases). This value might be underestimated because only the superior cerebellar artery was strongly observed through MRA or CTA examinations for the three blood vessels that supply the cerebellum, whereas the anterior inferior cerebellar and posterior inferior cerebellar arteries were weakly observed. Certain patients without a detectable vascular stenosis might have an occlusion in the anterior inferior cerebellar and posterior inferior cerebellar arteries based on the lesion distribution revealed by DWI. Therefore, we concluded that a stenosis in the vertebrobasilar extracranial segment was more common in patients with a cerebellar watershed infarction and that intracranial stenosis was more common in non-watershed infarction patients.

Our study showed that after hospital admission, patients with a cerebellar watershed infarction had a lower systolic blood pressure than non-watershed infarction patients but did not present with hypotension. This may have been observed because patients with a cerebellar watershed infarction have minor symptoms, patients with minor symptoms often cannot be admitted to the hospital, and temporary hypotension can cause watershed infarction. Compared with a non-watershed infarction, patients with a cerebellar watershed infarction have a more favorable prognosis. Among the cases included in our study, the vast majority of the cerebellar watershed infarction patients did not sustain disease progression after hospital admission, and no patients died. In contrast, symptoms in certain patients with a cerebellar non-watershed infarction had progressed one week after onset, and death was more common in patients with a posterior inferior cerebellar artery occlusion.

Causes for a brain infarction are complex and include the vascular factor, blood components, and brain perfusion. A brain infarction might result from a combined effect of these factors. Our study was limited because we analyzed the vascular factor and did not further classify cerebellar watershed infarction into internal watershed infarction and cortical watershed infarction given the small number of cases. Cortical watershed infarction caused by unstable plaque on the artery wall without stenosis was liable to neglected in our study. Our study showed that although patients with a cerebellar watershed infarction had mild symptoms and a good prognosis, they also had a high prevalence of stenosis in major blood vessels, especially the extracranial segment in the vertebral artery. Posterior circulation infarction has a high mortality rate, which suggests that once a cerebellar watershed infarction is identified, examination of the extracranial vessels should not be neglected, and early intervention should be performed to avoid arteriosclerosis and aggravation into a life-threatening cerebral infarction.

\section{Acknowledgements}

This study was supported by a grant from the Capital Health Research and Development of Special (Grant No. 2014-1-4092).

\section{Conflict of Interest}

The authors report no conflicts of interest.

\section{References}

[1] Amarenco, P., Kase, C.S., Rosengart, A., et al. (1993) Very Small (Border Zone) Cerebellar Infarcts. Distribution, Causes, Mechanisms and Clinical Features. Brain, 116, 161-186. http://dx.doi.org/10.1093/brain/116.1.161

[2] De Cocker, L.J., van Veluw, S.J., Fowkes, M., et al. (2013) Very Small Cerebellar Infarcts: Integration of Recent Insights into a Functional Topographic Classification. Cerebrovascular Diseases, 36, 81-87. http://dx.doi.org/10.1159/000353668

[3] Neurology Branch of Cerebrovascular Treatment Guidelines in Group of Acute Ischemic Stroke Writing Group of Chinese Medical Association (2010) China Guideline for Diagnosis and Treatment of Acute Ischemic Stroke. Chinese Journal of Neurology, 43, 146-153.

[4] Mangla, R., Kolar, B., Almast, J., et al. (2011) Border Zone Infarcts: Pathophysiologic and Imaging Characteristics. Radiographics, 31, 1201-1214. http://dx.doi.org/10.1148/rg.315105014

[5] Moustafa, R.R., Izquierdo-Garcia, D., Jones, P.S., et al. (2010) Watershed Infarcts in Transient Ischemic Attack/Minor Stroke with $>$ or $=50 \%$ Carotid Stenosis: Hemodynamic or Embolic? Stroke, 41, 1410-1416. http://dx.doi.org/10.1161/STROKEAHA.110.580415

[6] Momjian-Mayor, I. and Baron, J.C. (2005) The Pathophysiology of Watershed Infarction in Internal Carotid Artery 
Disease: Review of Cerebral Perfusion Studies. Stroke, 36, 567-577. http://dx.doi.org/10.1161/01.STR.0000155727.82242.e1

[7] Stump, D.A., Rogers, A.T., Hammon, J.W., et al. (1996) Cerebral Emboli and Cognitive Outcome after Cardiac Surgery. Journal of Cardiothoracic and Vascular Anesthesia, 10, 113-118. http://dx.doi.org/10.1016/S1053-0770(96)80186-8

[8] Bladin, C.F. and Chambers, B.R. (1994) Frequency and Pathogenesis of Hemodynamic Stroke. Stroke, 25, $2179-2182$. http://dx.doi.org/10.1161/01.STR.25.11.2179

[9] Masuda, J., Yutani, C., Ogata, J., et al. (1994) Atheromatous Embolism in the Brain: A Clinicopathologic Analysis of 15 Autopsy Cases. Neurology, 44, 1231-1237. http://dx.doi.org/10.1212/WNL.44.7.1231

[10] Wong, K.S., Gao, S., Chan, Y.L., et al. (2002) Mechanisms of Acute Cerebral Infarctions in Patients with Middle Cerebral Artery Stenosis: A Diffusion-Weighted Imaging and Microemboli Monitoring Study. Annals of Neurology, 52, 74-81. http://dx.doi.org/10.1002/ana.10250

[11] Caplan, L.R. and Hennerici, M. (1998) Impaired Clearance of Emboli (Washout) Is an Important Link between Hypoperfusion, Embolism, and Ischemic Stroke. Archives of Neurology, 55, 1475-1482. http://dx.doi.org/10.1001/archneur.55.11.1475

[12] Moriwaki, H., Matsumoto, M., Hashikawa, K., et al. (1997) Hemodynamic Aspect of Cerebral Watershed Infarction: Assessment of Perfusion Reserve Using Iodine-123-Iodoamphetamine SPECT. Journal of Nuclear Medicine, 38, 15561562.

[13] Lee, P.H., Bang, O.Y., Oh, S.H., et al. (2003) Subcortical White Matter Infarcts: Comparison of Superficial Perforating Artery and Internal Border-Zone Infarcts Using Diffusion-Weighted Magnetic Resonance Imaging. Stroke, 34, $2630-$ 2635. http://dx.doi.org/10.1161/01.STR.0000097609.66185.05

[14] Yong, S.W., Bang, O.Y., Lee, P.H., et al. (2006) Internal and Cortical Border-Zone Infarction: Clinical and DiffusionWeighted Imaging Features. Stroke, 37, 841-846. http://dx.doi.org/10.1161/01.STR.0000202590.75972.39 